\title{
Neonatal chest radiography: Influence of standard clinical protocols and radiographic equipment on pathology visibility and radiation dose using a neonatal chest phantom.
}

Introduction: Little is known about the variations in pathology visibility (PV) and their corresponding radiation dose values for neonatal chest radiography, between and within hospitals. Large variations in PV could influence the diagnostic outcome and the variations in radiation dose could affect the risk to patients. The aim of this study is to compare the PV and radiation dose for standard neonatal chest radiography protocols among a series of public hospitals.

Methods: A Gammex 610 neonatal chest phantom was used to simulate the chest region of neonates. Radiographic acquisitions were conducted on $17 \mathrm{X}$-ray machines located in eight hospitals, utilising their current neonatal chest radiography protocols. Six qualified radiographers assessed PV visually using a relative visual grading analysis (VGA). Signal to noise ratios (SNR) and contrast to noise ratios (CNR) were measured as a measure of image quality (IQ). Incident air kerma (IAK) was measured using a solid-state dosimeter.

Results: PV and radiation dose varied substantially between and within hospitals. For PV, the mean (range) VGA scores, between and within the hospitals, were 2.69 (2.00 to 3.50) and 2.73 (2.33 to 3.33), respectively. For IAK, the mean (range), between and within the hospitals, were 24.45 (8.11 to 49.94$) \mu \mathrm{Gy}$ and 34.86 (22.26 to 49.94$) \mu \mathrm{Gy}$, respectively.

Conclusion: Between and within participating hospitals there was wide variation in the visibility of simulated pathology and radiation dose (IAK).

Implications for practice: X-ray units with lower PV and higher doses require optimisation of their standard clinical protocols. Institutions which can offer acceptable levels of PV but with lower radiation doses should help facilitate national optimisation processes.

\section{Keywords}

Pathology visibility, image quality, radiation dose, dose optimisation, neonate chest radiography. 


\section{Introduction}

Optimisation of image quality (IQ) and radiation dose for neonatal chest X-ray images is a difficult task to undertake ${ }^{1,2}$. A particular problem for optimisation relates to the range of digital detectors and imaging equipment characteristics provided by manufacturers ${ }^{3}$. Differences are also likely to exist in the acquisition parameters that are applied to patients and the underlying reasons for this are likely to relate to radiographer / radiologist preferences as well as the inherent differences between the technologies. In turn these factors influence IQ and radiation dose. The small size, high tissue radiosensitivity and the inherently relatively low contrast for neonates presents additional challenges when attempting to perform chest radiography ${ }^{1,4-6}$. The Commission of the European Communities $(\mathrm{CEC})^{7}$ and the American College of Radiology (ACR $)^{8}$ have independently published two guidelines on paediatric radiographic imaging which refers to the chest region. Both guidelines have limitations. The CEC guideline is the most detailed with regard to paediatric radiography and it provides information on the image criteria necessary for accurate diagnosis as well as for the selection of optimal acquisition parameters. A major limitation of this report is that it was designed in an era of analogue film/screen systems. The ACR guideline is adapted for digital systems but it does not provide instructions regarding the optimal method for selecting acquisition parameters. No guidelines exist to identify an optimal protocol for digital neonatal chest radiography. Within the United Kingdom (UK), to the best of our knowledge, there is only one recent study that has been published ${ }^{9}$ investigating the variation in physical IQ (represented by low contrast detail detectability) and radiation dose between and within hospitals when undertaking paediatric chest radiography. However, this study has a limitation in that a physical phantom (CDRAD 2.0 phantom), with a uniform background, does not consider the impact of anatomical noise from human anatomy was used for IQ evaluation. It is worth noting that several studies have been undertaken to investigate the variations in IQ and dose for adult chest radiography within $\mathrm{UK}^{10,11}$.

Consequently, questions have arisen as to what extent do the expected variations in standard clinical protocols and radiographic equipment, between and within hospitals, impact on the resultant pathology visibility (PV) when undertaking neonatal chest radiography? Large variations in PV could influence the diagnostic accuracy. 
The aim of this study was to evaluate the differences in PV and radiation dose for neonatal chest radiographs, both between and within a series of hospitals using the Gammex 610 phantom.

\section{Materials and methods}

The study was undertaken using 17 X-ray machines located in eight UK National Health Service (NHS) hospitals within the North-west of England. Prior to starting the imaging process, quality control tests, based on the Institute of Physics and Engineering in Medicine (IPEM) report $91^{12}$, were undertaken for all X-ray machines included in this work. Tests typically include an assessment of radiation dose output variation with $\mathrm{kV}$, radiation dose output variation with $\mathrm{mA}$, radiation dose output variation with time and the overall reproducibility. Results demonstrated that the X-ray machines were working with expected tolerances.

\section{Phantom}

The Gammex 610 phantom (Gammex Inc., Middleton, WI) ${ }^{13}$ is an anthropomorphic neonatal chest phantom that simulates a $1-2 \mathrm{~kg}$ child. The structure of this phantom contains a torso, lungs, bronchial tree, spine, ribs and clavicle. It also includes clinically relevant IQ features for assessing resolution and noise, these are in the form of a lung with a simulated pneumothorax, pleural thickening and a lung with simulated hyaline membrane disease ${ }^{14}$. Pathological inserts are interchangeable and thus allow the simulation of a variety of scenarios, including normality.

\section{Image acquisition}

The Gammex 610 phantom was used to investigate the difference in PV, between and within the hospitals. Phantom images were acquired at each hospital/X-ray machine using their existing neonatal chest radiography protocols. The X-ray machine characteristics and their local examination acquisition factors are illustrated in Table 1. The Gammex phantom was positioned at the centre of radiation field and the X-ray field was collimated to the edges of the phantom. Resultant images were coded and transferred from each hospital/room in Digital Imaging and Communications in Medicine (DICOM) format for further analysis.

\section{Pathology visibility evaluation}

Ethical approval was granted from the University of Salford for this observer study (HSR161776). Gammex phantom images were assessed visually by six qualified diagnostic radiographers 
with clinical experience ranging from 5 to 18 years using a relative visual grading analysis (VGA) method and a 3 -point Likert scale ( $2=$ worse, $3=$ equal to, $4=$ better) using a bespoke display / observer response capture software ${ }^{15}$. This computer software is Java-based and presents images to observers in a random sequence. Images were viewed on two monitors, on the left monitor was a fixed reference image of average PV and on the right monitor the experimental images were displayed sequentially in a random order. The reference image was chosen by consensus opinion of two experienced clinicians (radiographers) who evaluate images as part of their normal clinical routine. The reference image had 'average' PV in comparison with the other images and the PV was represented by the visibility of a simulated pneumothorax and the respiratory distress syndrome, this was to ensure that all of the three Likert scale points were used in the evaluation 16,17. Each participant reviewed the images and responded to each question whilst making a comparison between the reference and experimental images. Observers decided whether PV was: worse, equal to or better than that of the reference image with the visibility of a simulated pneumothorax and respiratory distress syndrome being selected as criteria for assessing PV. All of the images were coded to ensure that the observers were blinded to the acquisition parameters and the hospital/X-ray machine.

For the PV evaluations, two 5 mega-pixel (2048 by 2560 pixels) display monitors (DOME E5, NDSsi, Santa Rosa, CA) were utilised. The monitors were calibrated to the DICOM grayscale standard display function $(\mathrm{GSDF})^{18}$ and the maximum luminance of the monitors was set at 500 cdlcm ${ }^{2}$ which represents the default value that is recommended by the manufacturer and the minimum luminance was set at $1 \mathrm{cdlcm}^{2}$. During image viewing, adjustment of image contrast, intensity and magnification by the observers was not permitted. The reason behind this was to ensure that any variations in PV were related to the quality of the image and not due to image manipulation. Also permitting the utilisation of image viewing tools, without restriction, might change the image appearances and introduce observational bias between observers. Ambient room lighting was below 8 lux. The final PV score for each image was calculated by taking the average (and the standard deviation [SD]) PV score for the six observers.

\section{Physical image quality measurements}

Physical image quality evaluation was undertaken using signal to noise ratios (SNR) and contrast to noise ratios (CNR) measurements. The average pixel values of four ROIs (Fig. 1) were 
considered as signal (circles 1 to 4), and noise was measured from the standard deviation of the background (circle 5). ImageJ (NIH, Bethesda, MD) was used to measure the SNR; all ROIs had the same area size $\left(1.37 \mathrm{~mm}^{2}\right)$ and were positioned in the same location on all images. SNR was calculated based on equation $(1)^{19}$ and CNR was calculated based on equation $(2)^{20,21}$. ROI positions were selected based on those reported in the literature ${ }^{22,23}$. The four ROIs (circles: 1, 2, 3 and 4) were only used for the calculation the SNR. For CNR, one ROI (circle 3) was selected. This ROI was considered more important clinically because any improvement in CNR for this region could influence the detection of lung disease.

$S N R=\frac{S}{\sigma_{b}}$

$C N R=\frac{S-B}{\sigma_{b}}$

Where:

$\mathrm{S}=$ average pixel values of the signal, $\mathrm{B}=$ average pixel values of the background, $\sigma_{b}=$ standard deviation of the background.

\section{Dosimetry}

A solid-state dosimeter (RaySafe X2, Unfors Ray Safe AB, Billdal, Sweden) was used to measure the incident air kerma (IAK) at the entry point of the X-ray beam central ray on the anterior surface of the phantom. Four exposures were made for each protocol - one to produce the image and three to record a mean dose, along with the SD to reduce the random error.

\section{Data analysis}

SPSS version 22.0 (IBM Inc, Armonk, New York, US) was used for analysing the data. The Shapiro-Wilk test was used to examine the normality of the data to determine the appropriate correlation statistic between PV and IAK and also PV and SNR / CNR. The strength of the correlation (r) was interpreted based on the literature ${ }^{24,25}$, in which $r=0.10-0.29$ (small), $r=0.30$ 0.49 (medium), and $\mathrm{r}=0.50-1.0$ (large). For investigating the inter-observer variability in the PV evaluations, an inter-class correlation coefficient (ICC) was used. ICC values less than 0.50 
indicate poor reliability, values from 0.50 to 0.75 indicate moderate reliability, values from 0.75 to 0.9 indicate good reliability and finally values greater than 0.90 indicates excellent reliability ${ }^{26,27}$.

\section{Results}

IAK, PV, SNR and CNR data are presented as a series of bar charts, while the IAK values are also presented as a dashed line against the corresponding PV values within the same graph. Hospitals and X-ray machines were coded: the letter $(\mathrm{H})$ refers to the hospital and the letter $(\mathrm{X})$ refers to the X-ray machine i.e. Hospital 1, X-ray machine 1 would be H1X1. Figs. 2 and 3 compare the IAK and PV, between and within hospitals, respectively; Fig. 4 compares the PV scores against the corresponding IAK values, between and within hospitals. Figs. 5 and 6 display the variation in SNR and CNR, both between and within the hospitals, respectively.

IAK values between the hospitals ranged from 8.1 to $49.9 \mu \mathrm{Gy}$ with a $144.1 \%$ difference. The range within the hospitals was smaller, 22.3 to $49.9 \mu \mathrm{Gy}$ with a $76.7 \%$ difference. The percentage difference was calculated based on the following equation: -

$$
\frac{|V 1-V 2|}{(V 1+V 2) / 2} * 100
$$

Where $\mathrm{V} 1$ is value 1 and $\mathrm{V} 2$ is value 2.

The PV scores between the hospitals ranged from 2.00 to 3.50 with a $54.5 \%$ difference. Within the hospitals the range was smaller, 2.3 to 3.3 with a $35.3 \%$ difference. CNR values between the hospitals ranged from 7.7 to 33.2 with a $125.1 \%$ difference. The within hospital range was smaller, 10 to 33.2 with a $107.4 \%$ difference. Between hospitals SNR ranged from 31.0 to 98.0 with a $102.7 \%$ difference. Within hospitals CNR varied from 44.6 to 98.0 with a smaller (74.9\%) difference.

A large variation was observed in milliampere seconds (mAs), from 0.63 to $2 \mathrm{mAs}$, between and within hospitals, and the variations in the range of kilovoltage peak $(\mathrm{kVp})$ and source to image detector distance (SID) were 60 to $64.5 \mathrm{kVp}$ and 110 to $135 \mathrm{~cm}$, respectively. Finally, additional filtration was utilised by only two hospitals, and there was no consistency between the X-ray machines in the focal spot type used. 
A moderate, non-significant, correlation between PV and IAK was displayed ( $\mathrm{r}=0.46 ; \mathrm{P}=0.06)$. Moderate inter-observer agreement between the observers was observed (ICC $=0.54 ; 95 \%$ CI 0.17 to 0.80). Non-statistically significant correlations between PV and SNR and PV and CNR were observed to be $\mathrm{r}=0.27(\mathrm{P}=0.29)$ and $\mathrm{r}=0.13(\mathrm{P}=0.61)$, respectively.

\section{Discussion}

Our study identified a difference in PV and IAK, between and within centres. Regarding the visual evaluation of PV (Fig. 4), the possible cause of PV variation is related to the differences in acquisition parameters, techniques and the type of image processing used between and within centres. Furthermore, the differences in radiation dose among X-ray machines may also be related to the X-ray machine age with equipment ageing impacting on radiation output and consequently PV. A large variation was observed in mAs values (Table 1) and this can have a high impact on the differences of PV between and within hospitals. High mAs values increase the number of the photons hitting the image detector and this causes an increase in the SNR and a subsequent increase

PV, and vice versa. From the literature, it has been demonstrated that there is a direct positive relationship between $\mathrm{mAs}$ and IQ ${ }^{28-30}$. Also, the variations in SID and $\mathrm{kVp}$ values (Table 1) can also have an influence on the variation in PV between and within the centres. Both the high SID and low $\mathrm{kVp}$ will reduce the number of the photons hitting the image detector and this will reduce the SNR with a subsequent decrease in PV scores and vice versa. Another factor that can contributes to the variation in PV between and within the centres are the differences in the characteristics of the X-ray imaging systems, since within practice there is both computed radiography (CR) and digital radiography (DR) systems. Many studies indicated that the performance of DR systems is superior to that of CR systems for PV, under similar dose levels ${ }^{31,32}$.

The PV evaluation in our study indicated only the variation in visibility of the simulated pneumothorax and respiratory distress syndrome, between and within centres, and there was no detection task (simulated pathology detection) within our study. The clinical influence of the observed differences in PV, especially for the centres with the three lowest values of PV should be considered in future work to help maximise pathology detection performance. The variation in PV might influence the detectability of subtle pathology in clinical practice. Further studies are required to investigate to what extent this variation in PV might influence pathology detectability. 
If the low values of PV observed in this study do not influence the diagnostic accuracy regarding pathology detection, the radiation dose can be minimised considerably between and within institutions. The acquisition parameters for neonatal chest radiographs should be selected based on the clinical indications for achieving acceptable IQ for diagnosis. It could be that an imaging protocol and its resultant radiation dose is optimal for general IQ evaluations but it might be not acceptable for detectability of a small subtle lung pathology.

In terms of radiation dose, similar to $\mathrm{PV}$, the variations in radiation dose are likely related to the differences in acquisition parameters used between and within centres. For instance, previous studies reported that there is a direct relationship between the mAs, number of X-ray photons produced and the radiation dose received by the patients /phantoms ${ }^{33,34}$. Another example, is the influence of acquisition parameters on the radiation dose such as the variation in SID and $\mathrm{kVp}$ used, between and within centres (Table 1), both a high SID and low $\mathrm{kVp}$ will reduce IAK and vice versa. Moreover, additional filtration is also considered to be a factor for decreasing radiation dose in this study. Utilising additional beam filtration minimises low X-ray energies and increases the penetration energy, this then leads to a reduction in the absorbed dose for the imaged object ${ }^{35-}$ 37. Another reason that can causes the variation in PV and dose is the differences in imaging equipment, within practice there is both CR and DR systems. For instance, the CR system requires more radiation in comparison with DR to generate a similar IQ. Possible DR dose decreases of up to $75 \%$ have been reported in comparison with $\mathrm{CR}^{32,38-40}$.

A weak non-significant correlation was found between PV and SNR and PV and CNR ( $r=0.27$; $\mathrm{P}=0.29)$ and $(\mathrm{r}=0.13 ; \mathrm{P}=0.61)$, respectively. This indicates that these physical measures might not be particularly good indicators when assessing visual PV on neonatal chest radiographs. The reason behind this is that they have high sensitivity to changes in quantum noise and pixel values than that of the human eye, which cannot easily detect such subtle changes. A moderate correlation between IAK and PV is considered an indication that high PV is not necessarily related to a high radiation dose and the radiographic imaging protocols are not well optimised. Overall the results provide evidence that there is potential for further optimisation by decreasing radiation exposure without necessarily degrading PV. This is especially necessary for the X-ray machines observed with low PV and high radiation dose, optimisation their imaging protocols could increase the pathology detection performance in clinical practice and also minimise radiation exposure and risk 
to patients. Although our study has been conducted on a small sample of X-ray units, it is reasonable to speculate that our findings would be applicable to neonatal chest imaging generally. The reason behind this is because neonatal chest radiography is commonly a difficult examination in which to achieve IQ and dose optimisation, and such differences in PV and dose are likely exist in other hospitals and imaging systems which use mobile X-ray machines since the variations in standard clinical imaging protocols and imaging systems performance still exist. With this in mind, for dose and PV, we suggest that neonatal chest imaging should be investigated in all institutions to ensure conditions are optimal.

Our study focused on neonates in view of their higher sensitivity to radiation when compared with adults $^{41}$. We further focused on chest radiographs rather than other anatomical areas since it is a common examination for neonates ${ }^{42}$. The Gammex phantom was used for PV evaluations, this appears to be well justified since this phantom is considered the best commercially available phantom for simulating a neonate's chest ${ }^{19,28,43-45}$. A limitation of this study relates to the type of imaging equipment used for neonatal examinations since these were predominantly stationary Xray machines while neonatal imaging is often undertaken with mobile X-ray machines; therefore, further work using our method should be undertaken with a greater range of mobile X-ray machines. Our work should also be extended to include a larger sample of X-ray machines.

\section{Conclusion}

Findings from our study show differences in IAK and PV between and within hospitals, but the difference within hospitals was lower. This is likely to be related to 1) variations in acquisition parameters and techniques; 2) technical characteristics of the imaging equipment. A moderate correlation $(\mathrm{r}=0.46)$ between IAK and PV was observed and this is a further indication that high PV is not necessarily related to a high radiation dose. The results indicate that the neonatal chest

X-ray images should be investigated in all institutions to ensure conditions are optimal. The X-ray units with lower PV and higher dose are required optimisation their standard clinical protocols to reducing radiation risk to patients and increasing pathology detection performance. Overall, the results provide evidence that there is potential for more optimisation by decreasing radiation exposure without necessarily degrading PV. 
On the other hand, the findings can be used as a baseline for any future local and national reference doses, and the data from this study could be useful as a baseline for any new surveys and studies on the optimisation of PV and radiation does for digital neonatal chest radiography.

\section{References}

1. Kim T-H., Ryu J-H., Jeong C-W., Jun HY., Heo D-W., Lee SH., et al. Reduced radiation dose and improved image quality using a mini mobile digital imaging system in a neonatal intensive care unit. Clin Imaging 2017;42:165-71. Doi: 10.1016/j.clinimag.2016.12.004.

2. Conradie A., Herbst CP. Evaluating the effect of reduced entrance surface dose on neonatal chest imaging using subjective image quality evaluation. Phys Medica 2016;32(10):136874. Doi: 10.1016/j.ejmp.2016.07.005.

3. Kroft LJM., Veldkamp WJH., Mertens BJA., Boot M V., Geleijns J. Comparison of eight different digital chest radiography systems: Variation in detection of simulated chest disease. Am J Roentgenol 2005;185(2):339-46. Doi: 10.2214/ajr.185.2.01850339.

4. Armpilia CI., Fife IAJ., Croasdale PL. Radiation dose quantities and risk in neonates in a special care baby unit. Br J Radiol 2002;75(895):590-5. Doi: 10.1259/bjr.75.895.750590.

5. de González AB., Darby S. Risk of cancer from diagnostic X-rays: estimates for the UK and 14 other countries. Lancet 2004;363(9406):345-51. Doi: 10.1016/S01406736(04)15433-0.

6. Guo H., Liu W., He X., Zhou X., Zeng Q., Li B. Optimizing Imaging Quality and Radiation Dose by the Age-Dependent Setting of Tube Voltage in Pediatric Chest Digital Radiography. Korean J Radiol 2013;14(1):126. Doi: 10.3348/kjr.2013.14.1.126.

7. Commission of the European Communities (CEC). European Guidelines on Quality Criteria for Diagnostic Radiographic Images:(EUR 16260 EN)., Luxembourg: CEC; 1996.

8. American College of Radiology. ACR-SPR practice parameter for the performance of chest radiography, 2014.

9. Al-Murshedi S., Hogg P., Meijer A., Erenstein H., England A. Comparative analysis of radiation dose and low contrast detail detectability using routine paediatric chest radiography protocols. Eur J Radiol 2019;113(September 2018):198-203. Doi: 10.1016/j.ejrad.2019.02.017.

10. Al-Murshedi S., Hogg P., England A. Relationship between body habitus and image quality and radiation dose in chest X-ray examinations: A phantom study. Phys Medica 2019;57(December 2018):65-71. Doi: 10.1016/j.ejmp.2018.12.009.

11. Al-Murshedi S., Hogg P., Lanca L., England A. A novel method for comparing radiation 
dose and image quality, between and within different X-ray units in a series of hospitals. $J$ Radiol Prot 2018. Doi: 10.1088/1361-6498/aae3fa.

12. Institute of Physics and Engineering in Medicine. IPEM report 91: recommended standards for the routine performance testing of diagnostic x-ray imaging systems, York: IPEM; 2005.

13. Anthropomorphic Neonatal Chest Phantom. Https://Www.Sunnuclear.Com/Shopgammex/Anthropomorphic-Neo-Natal-ChestPhantom.

14. Dewerd L.A KM. The Phantoms of Medical and Health Physics, New York.: Springer; 2014.

15. Hogg, P. and Blindell P. Software for image quality evaluation using a forced choice method. In United Kingdom radiological conference (p. 139). British Institute of Radiology, Manchester/London, UK., 2012.

16. Allen E., Hogg P., Ma WK., Szczepura K. Fact or fiction: An analysis of the 10 kVp 'rule' in computed radiography. Radiography 2013;19(3):223-7. Doi: 10.1016/j.radi.2013.05.003.

17. Ma WK., Hogg P., Tootell A., Manning D., Thomas N., Kane T., et al. Anthropomorphic chest phantom imaging - The potential for dose creep in computed radiography. Radiography 2013;19(3):207-11. Doi: 10.1016/j.radi.2013.04.002.

18. The Royal College of Radiologists. Picture archiving and communication systems (PACS) and guidelines on diagnostic display devices, 2012.

19. Smans K., Struelens L., Smet M., Bosmans H., Vanhavere F. Cu filtration for dose reduction in neonatal chest imaging. Radiat Prot Dosimetry 2010;139(1-3):281-6. Doi: 10.1093/rpd/ncq061.

20. Hampel JR., Pascoal A. Comparison and optimization of imaging techniques in suspected physical abuse paediatric radiography. Br J Radiol 2018;91(December 2017):20170650. Doi: 10.1259/bjr.20170650.

21. Jiang X., Baad M., Reiser I., Feinstein KA., Lu Z. Effect of comfort pads and incubator design on neonatal radiography. Pediatr Radiol 2016;46(1):112-8. Doi: 10.1007/s00247015-3450-5.

22. Lin Y., Luo H., Dobbins JT., Page McAdams H., Wang X., Sehnert WJ., et al. An imagebased technique to assess the perceptual quality of clinical chest radiographs. Med Phys 2012;39(11):7019-31. Doi: 10.1118/1.4760886.

23. Mraity H., England A., Akhtar I., Aslam A., De Lange R., Momoniat H., et al. Development and validation of a psychometric scale for assessing PA chest image quality: A pilot study. Radiography 2014;20(4):312-7. Doi: 10.1016/j.radi.2014.03.007.

24. Filed A. Discovering statistics using SPSS statistics, Third edit, Londan: Sage publications Ltd; 2009.

25. Cohen J. Statistical power analysis for the behavioral sciences.Hilsdale." NJ: Lawrence 
Earlbaum Associates 2, 1988.

26. Koo TK., Li MY. A Guideline of Selecting and Reporting Intraclass Correlation Coefficients for Reliability Research. J Chiropr Med 2016;15(2):155-63. Doi: 10.1016/j.jcm.2016.02.012.

27. Portney L., Watkins M. Foundations of clinical research: applications to practice.New Jersey: Prentice Hall, 2000.

28. Precht H., Tingberg A., Waaler D., Outzen CB. New Developed DR Detector Performs Radiographs of Hand, Pelvic and Premature Chest Anatomies at a Lower Radiation Dose and/or a Higher Image Quality. J Digit Imaging 2014;27(1):68-76. Doi: 10.1007/s10278013-9635-2.

29. De Hauwere A., Bacher K., Smeets P., Verstraete K., Thierens H. Analysis of image quality in digital chest imaging. Radiat Prot Dosimetry 2005;117(1-3):174-7. Doi: 10.1093/rpd/nci748.

30. Saito K., Hiramoto S., Gomi T., Muramoto N., Seki M., Tsukimura K., et al. Evaluation of chest and abdominal exposure dose appropriate for a digital image reader system incorporating a columnar-crystal structured phosphor plate and a contrast-detail phantom. Radiol Phys Technol 2008;1(2):238-43. Doi: 10.1007/s12194-008-0035-0.

31. Bacher K., Smeets P., Bonnarens K., De Hauwere A., Verstraete K., Thierens H. Dose Reduction in Patients Undergoing Chest Imaging: Digital Amorphous Silicon Flat-Panel Detector Radiography Versus Conventional Film-Screen Radiography and Phosphor-Based Computed Radiography. Am J Roentgenol 2003;181(4):923-9. Doi: 10.2214/ajr.181.4.1810923.

32. Hamer OW., Völk M., Zorger Z., Feuerbach S., Strotzer M. Amorphous silicon, flat-panel, X-ray detector versus storage phosphor-based computed radiography: contrast-detail phantom study at different tube voltages and detector entrance doses. Invest Radiol 2003;38(4):212-20. Doi: 10.1097/01.RLI.0000057032.41715.15.

33. Sun Z., Lin C., Tyan Y., Ng KH. Optimization of chest radiographic imaging parameters: A comparison of image quality and entrance skin dose for digital chest radiography systems. Clin Imaging 2012;36(4):279-86. Doi: 10.1016/j.clinimag.2011.09.006.

34. Seeram E., Davidson R., Bushong S., Swan H. Optimizing the Exposure Indicator as a Dose Management Strategy in Computed Radiography. Radiol Technol 2016;87(4):380-91.

35. Brosi P., Stuessi A., Verdun FR., Vock P., Wolf R. Copper filtration in pediatric digital Xray imaging: its impact on image quality and dose. Radiol Phys Technol 2011;4(2):148-55. Doi: 10.1007/s12194-011-0115-4.

36. Ekpo EU., Hoban AC., McEntee MF. Optimisation of direct digital chest radiography using $\mathrm{Cu}$ filtration. Radiography 2014;20(4):346-50. Doi: 10.1016/j.radi.2014.07.001.

37. Hamer OW., Völk M., Zorger N., Borisch I., Büttner R., Feuerbach S., et al. Contrast-detail phantom study for $\mathrm{X}$-ray spectrum optimization regarding chest radiography using a cesium iodide-amorphous silicon flat-panel detector. Invest Radiol 2004;39(10):610-8. Doi: 00004424-200410000-00004 [pii]. 
38. McEntee M., Frawley H., Brennan PC. A comparison of low contrast performance for amorphous Silicon/caesium iodide direct radiography with a computed radiography: A contrast detail phantom study. Radiography 2007;13(2):89-94. Doi: 10.1016/j.radi.2006.01.004.

39. Jansson M., Geijer H., Persliden J., Andersson T. Reducing dose in urography while maintaining image quality - a comparison of storage phosphor plates and a flat-panel detector. Eur Radiol 2006;16(1):221-6. Doi: 10.1007/s00330-005-2772-3.

40. Fischbach F., Ricke J., Freund T., Werk M., Spors B., Baumann C., et al. Flat panel digital radiography compared with storage phosphor computed radiography: assessment of dose versus image quality in phantom studies. Invest Radiol 2002;37(11):609-14. Doi: 10.1097/01.RLI.0000031078.14395.E4.

41. Raissaki MT. Pediatric radiation protection. Eur Radiol Suppl 2004;14(S1):74-83. Doi: 10.1007/s10406-004-0011-7.

42. Joarder R., Crundwell N. Chest X-Ray in Clinical Practice, London: Springer London; 2009.

43. Singh VH., Pradhan H. Neonatal chest radiography - Comparing image quality and dose for contact-techniques vs. under-tray techniques. Radiogr Open 2015;2(1):65. Doi: 10.7577/radopen.1530.

44. Cohen MD., Markowitz R., Hill J., Huda W., Babyn P., Apgar B. Quality assurance: A comparison study of radiographic exposure for neonatal chest radiographs at 4 academic hospitals. Pediatr Radiol 2012;42(6):668-73. Doi: 10.1007/s00247-011-2290-1.

45. Smet MH., Breysem L., Mussen E., Bosmans H., Marshall NW., Cockmartin L. Visual grading analysis of digital neonatal chest phantom X-ray images: Impact of detector type, dose and image processing on image quality. Eur Radiol 2018;28(7):2951-9. Doi: 10.1007/s00330-017-5301-2.

\section{Figures descriptions:}

Fig. 1. A Gammex phantom image illustrating the position of ROIs (circles:1, 2, 3 and 4) and background (circle: 5).

Fig. 2. A bar chart displaying the variability of incident air kerma (IAK) values across the different $\mathrm{X}$-ray machines between/within hospitals. The error bars in this chart represents the standard deviation (SD) in the incident air kerma (IAK) obtained from measuring the three repeat radiation exposures. $\mathrm{H}=$ hospital; $\mathrm{X}=\mathrm{X}$-ray machine.

Fig. 3. A bar chart displaying the variability of pathology visibility (PV) values across the different $\mathrm{X}$-ray machines between/within hospitals. The error bars in this chart represent the standard 
deviation (SD) in PV obtained from the visual evaluations by observers. $\mathrm{H}=$ hospital; $\mathrm{X}=\mathrm{X}$-ray machine.

Fig. 4. A comparison of measured pathology visibility (PV) against the incident air kerma (IAK) between/within hospitals. The error bars in this chart represent the standard deviation (SD) in PV obtained from the visual evaluations by observers. The dashed line represents the average value of the IAK that resulted from measuring three repeated radiation exposures and although connected does not imply a relationship between machines/hospitals. $\mathrm{H}=$ hospital; $\mathrm{X}=\mathrm{X}$-ray machine.

Fig. 5. A bar chart displaying the variability of signal to noise ratios (SNR) values across the different $\mathrm{X}$-ray machines between/within hospitals. $\mathrm{H}=$ hospital; $\mathrm{X}=\mathrm{X}$-ray machine.

Fig. 6. A bar chart displaying the variability of contrast to noise ratios (CNR) values across the different $\mathrm{X}$-ray machines between/within hospitals. $\mathrm{H}=$ hospital; $\mathrm{X}=\mathrm{X}$-ray machine.

\section{Tables descriptions:}

Table 1. A summary of X-ray machine types, examination techniques and acquisition parameters used for neonatal chest radiography. 


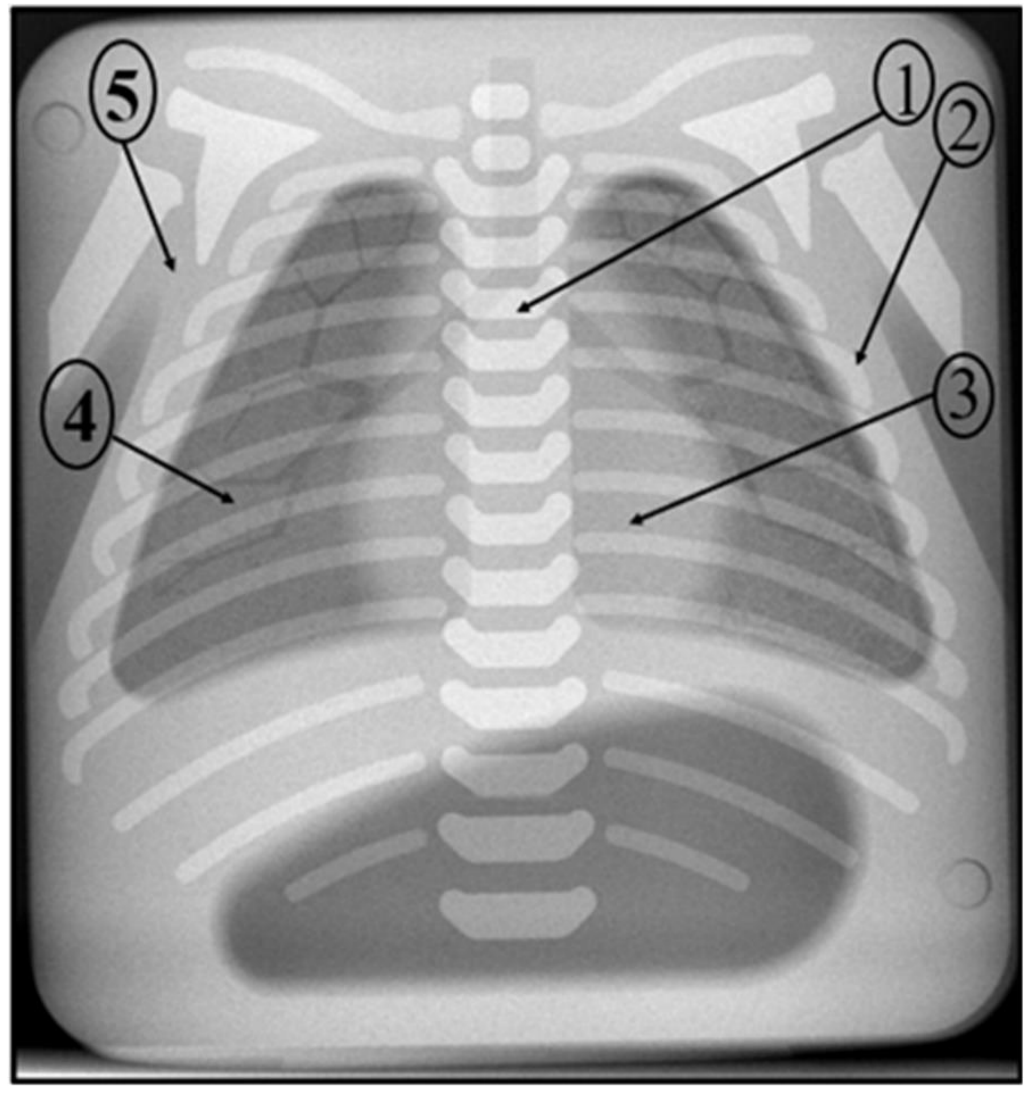

Fig. 1. A Gammex phantom image illustrating the position of ROIs (circles:1, 2, 3 and 4) and background (circle: 5 ).

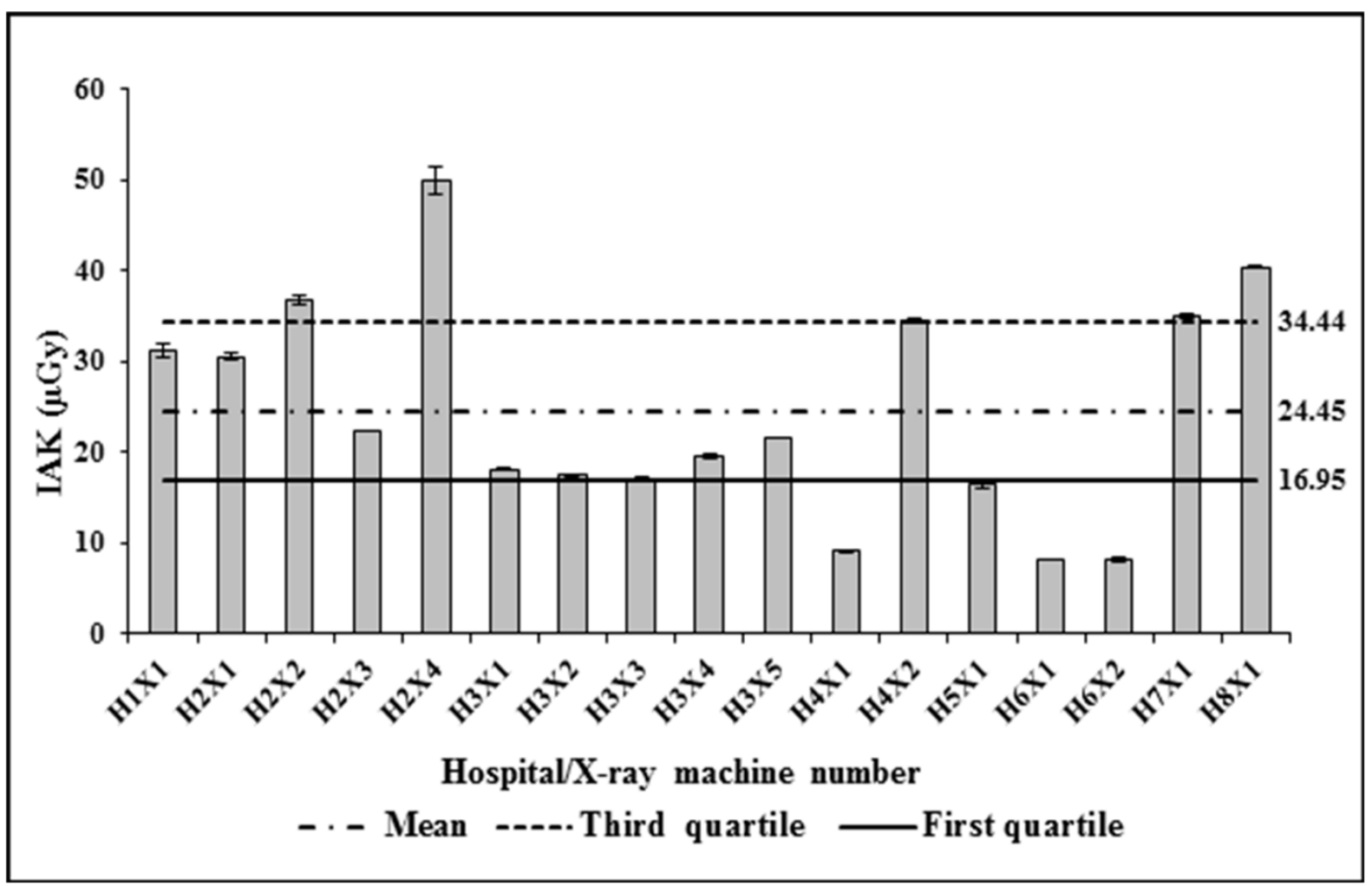


Fig. 2. A bar chart displaying the variability of incident air kerma (IAK) values across the different X-ray machines between/within hospitals. The error bars in this chart represents the standard deviation (SD) in the incident air kerma (IAK) obtained from measuring the three repeat radiation exposures. $\mathrm{H}=$ hospital; $\mathrm{X}=\mathrm{X}$-ray machine.

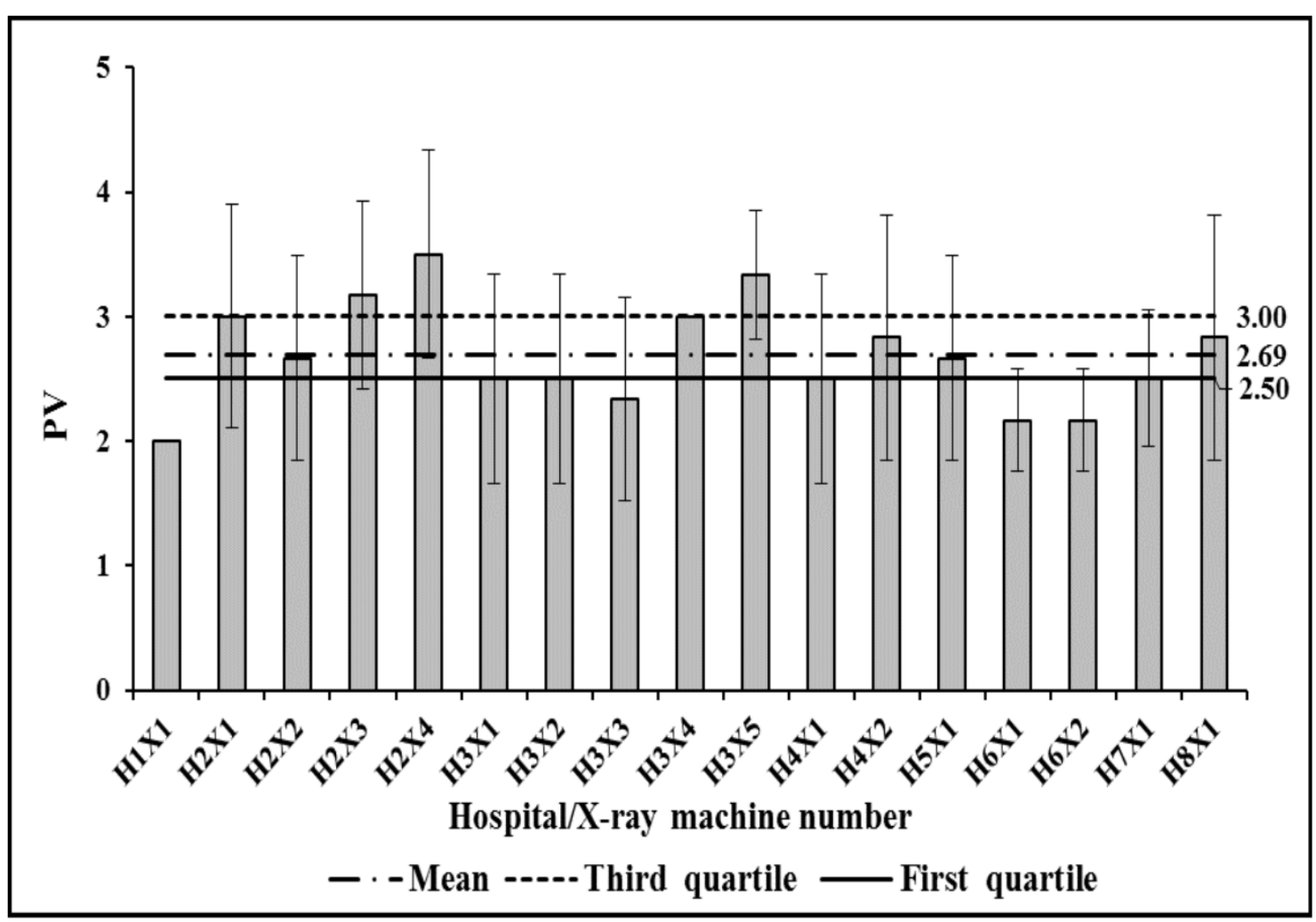

Fig. 3. A bar chart displaying the variability of pathology visibility (PV) values across the different X-ray machines between/within hospitals. The error bars in this chart represent the standard deviation (SD) in PV obtained from the visual evaluations by observers. $\mathrm{H}=$ hospital; $\mathrm{X}=\mathrm{X}$-ray machine. 


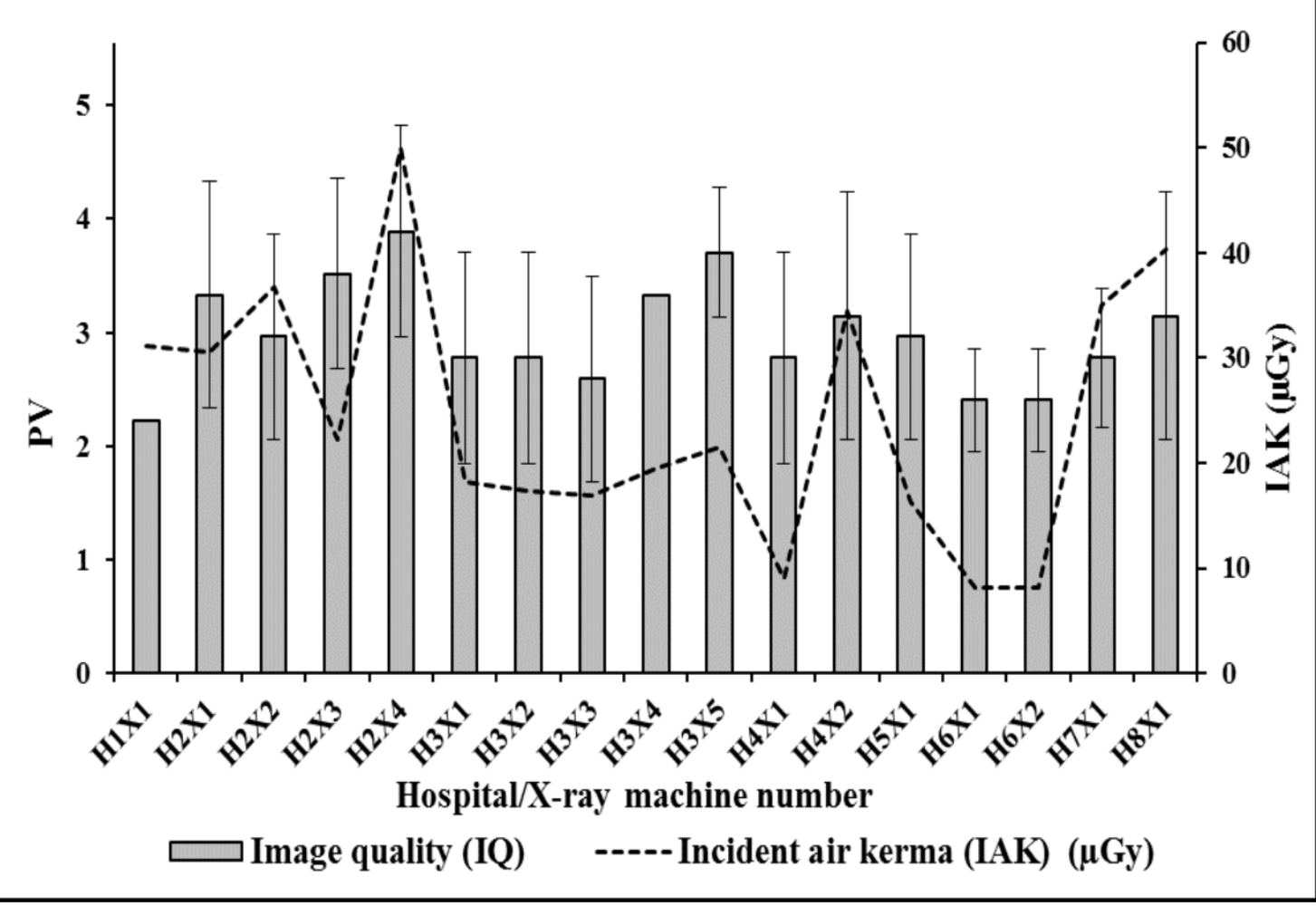

Fig. 4. A comparison of measured pathology visibility (PV) against the incident air kerma (IAK) between/within hospitals. The error bars in this chart represent the standard deviation (SD) in PV obtained from the visual evaluations by observers. The dashed line represents the average value of the IAK that resulted from measuring three repeated radiation exposures and although connected does not imply a relationship between machines/hospitals. $\mathrm{H}=$ hospital; $\mathrm{X}=\mathrm{X}$-ray machine. 


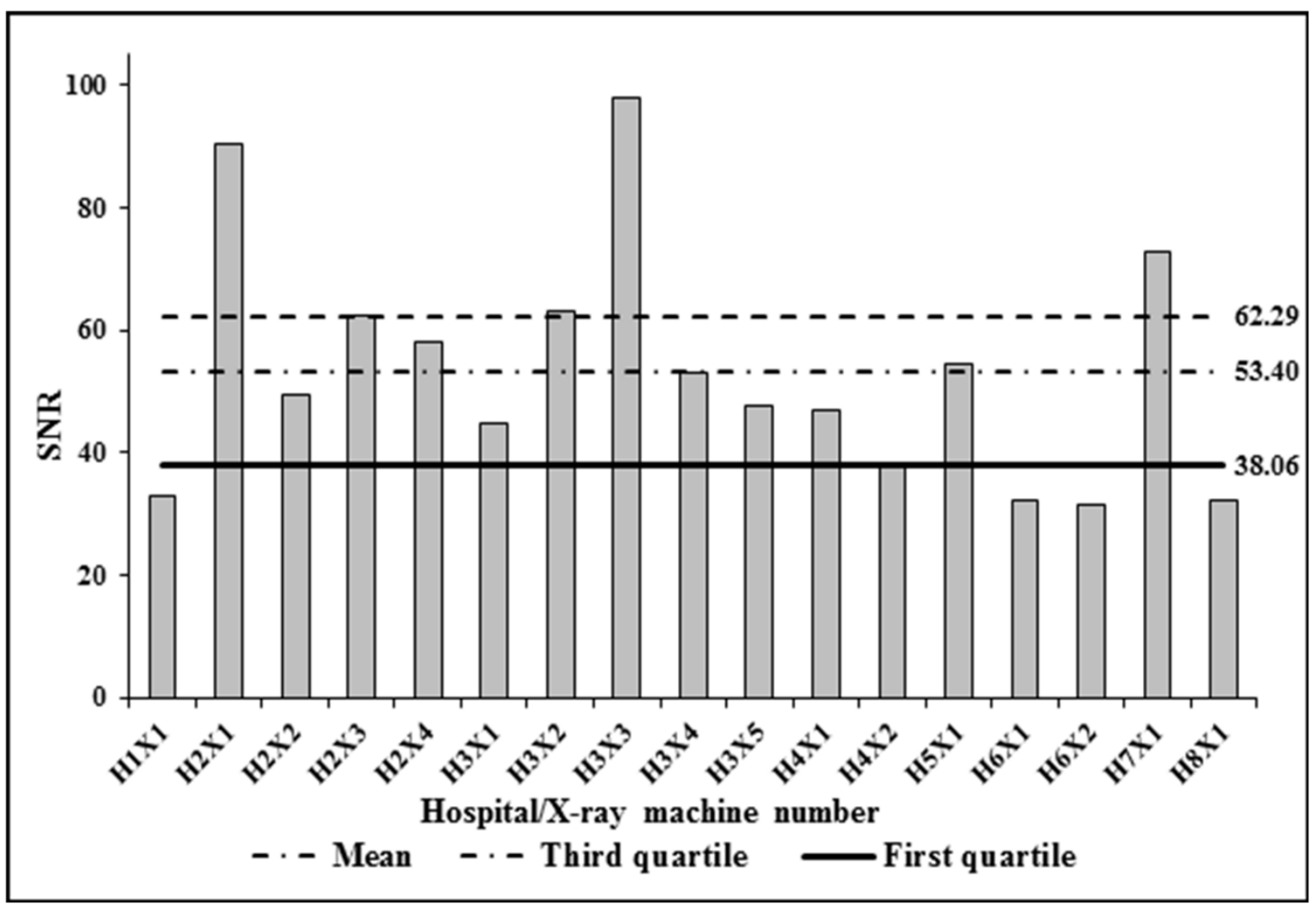

Fig. 5. A bar chart displaying the variability of signal to noise ratios (SNR) values across the different $\mathrm{X}$-ray machines between/within hospitals. $\mathrm{H}=$ hospital; $\mathrm{X}=\mathrm{X}$-ray machine. 


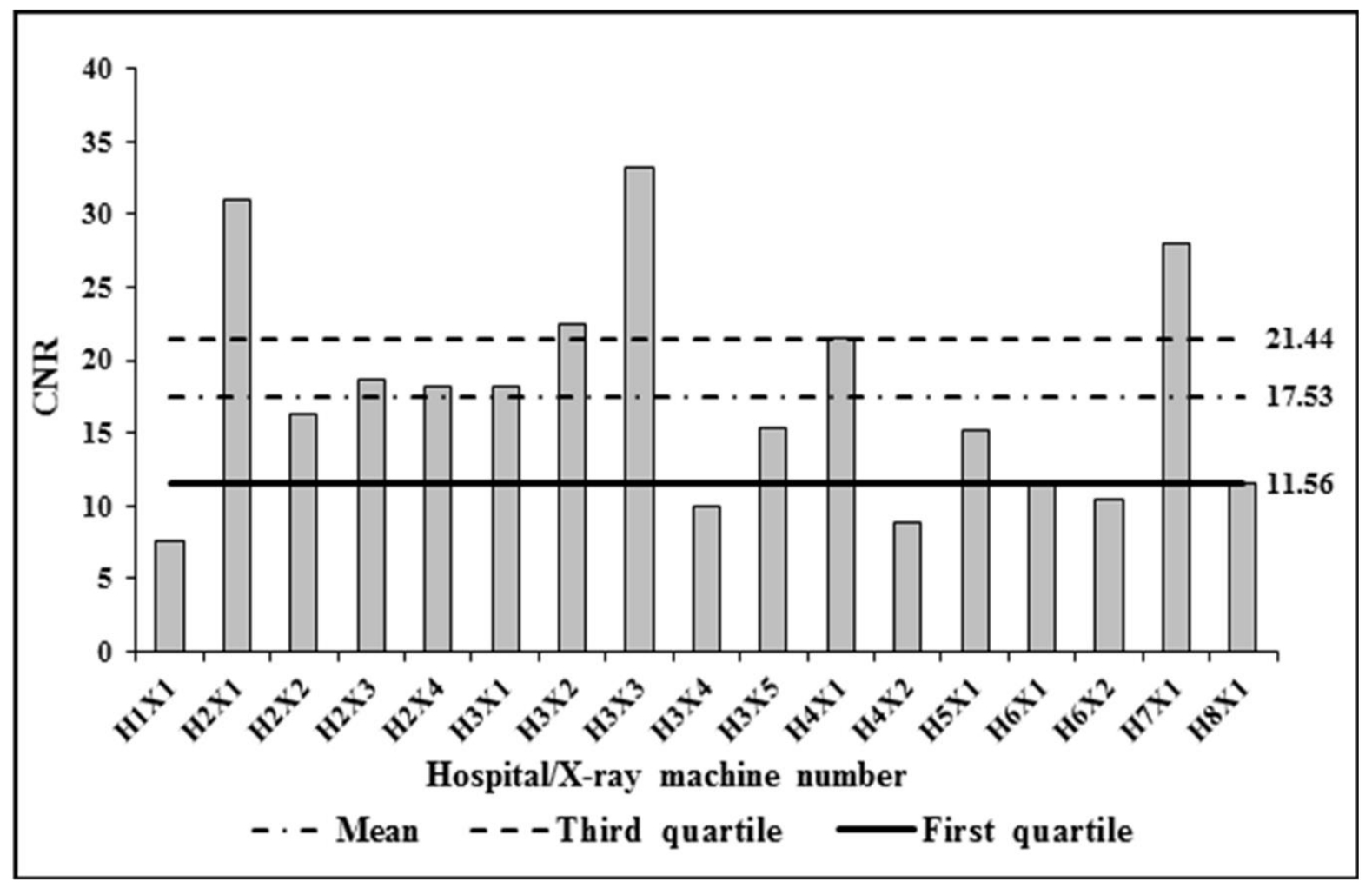

Fig. 6. A bar chart displaying the variability of contrast to noise ratios (CNR) values across the different X-ray machines between/within hospitals. $\mathrm{H}=$ hospital; $\mathrm{X}=\mathrm{X}$-ray machine. 
Table 1. A summary of X-ray machine types, examination techniques and acquisition parameters used for neonatal chest radiography.

\begin{tabular}{|c|c|c|c|c|c|c|c|c|c|c|c|c|}
\hline $\begin{array}{c}\text { Hospital } \\
\text { number }\end{array}$ & $\begin{array}{c}\text { X-ray } \\
\text { machine } \\
\text { number }\end{array}$ & $\begin{array}{c}\text { Machine } \\
\text { type }\end{array}$ & $\begin{array}{l}\text { Detector } \\
\text { Type }\end{array}$ & $\begin{array}{c}\text { Machine } \\
\text { Manufacturer }\end{array}$ & $\begin{array}{c}\text { Detector } \\
\text { Manufacturer }\end{array}$ & $\begin{array}{c}\text { Exposure } \\
\text { control }\end{array}$ & Grid & $\begin{array}{l}\text { Additional } \\
\text { filtration } \\
\text { (mm) }\end{array}$ & $\begin{array}{c}\text { Focal } \\
\text { spot }\end{array}$ & $\begin{array}{l}\text { SID }^{h} \\
(\mathbf{c m})\end{array}$ & $k V p$ & mAs \\
\hline 1 & 1 & Static & $\mathrm{IDR}^{\mathrm{a}}$ & Philips $^{\mathrm{d}}$ & Philips & Manual & No & No & Broad & 135 & 60 & 1.25 \\
\hline \multirow[t]{4}{*}{2} & 1 & Mobile & IDR & Carestream $^{\mathrm{e}}$ & Carestream & Manual & No & No & Broad & 115 & 64 & 0.80 \\
\hline & 2 & Static & IDR & Siemens ${ }^{\mathrm{f}}$ & Siemens & Manual & No & No & Broad & 115 & 68 & 1.00 \\
\hline & 3 & Static & $\mathrm{IDR}^{\mathrm{b}}$ & Carestream & Carestream & Manual & No & No & Broad & 130 & 60 & 1.20 \\
\hline & 4 & Static & IDR & Samsung ${ }^{g}$ & Samsung & Manual & No & No & Broad & 115 & 63 & 2.00 \\
\hline \multirow[t]{5}{*}{3} & 1 & Static & IDR & Siemens & Siemens & Manual & No & $0.1 \mathrm{Cu}$ & Fine & 115 & 64.5 & 1.40 \\
\hline & 2 & Static & IDR & Siemens & Siemens & Manual & No & $0.1 \mathrm{Cu}$ & Fine & 115 & 64.5 & 1.40 \\
\hline & 3 & Static & IDR & Siemens & Siemens & Manual & No & $0.1 \mathrm{Cu}$ & Fine & 115 & 64.5 & 1.40 \\
\hline & 4 & Static & IDR & Siemens & Siemens & Manual & No & $0.1 \mathrm{Cu}$ & Fine & 115 & 64.5 & 1.40 \\
\hline & 5 & Static & IDR & Siemens & Siemens & Manual & No & $0.1 \mathrm{Cu}$ & Fine & 115 & 64.5 & 1.40 \\
\hline \multirow[t]{2}{*}{4} & 1 & Static & DDR & Philips & Philips & Manual & No & $1 \mathrm{Al}+0.1 \mathrm{Cu}$ & Fine & 110 & 60 & 1.00 \\
\hline & 2 & Static & $\mathrm{CR}^{\mathrm{c}}$ & Philips & Carestream & Manual & No & No & Fine & 110 & 60 & 1.00 \\
\hline 5 & 1 & Static & $\mathrm{CR}$ & Siemens & Carestream & Manual & No & No & Fine & 120 & 60 & 0.63 \\
\hline \multirow[t]{2}{*}{6} & 1 & Static & DDR & Philips & Philips & Manual & No & No & Broad & 135 & 60 & 1.20 \\
\hline & 2 & Static & DDR & Philips & Philips & Manual & No & No & Broad & 135 & 60 & 1.20 \\
\hline 7 & 1 & Static & IDR & Carestream & Carestream & Manual & No & No & Broad & 115 & 60 & 1.20 \\
\hline 8 & 1 & Static & $\mathrm{CR}$ & Siemens & Carestream & Manual & No & No & Broad & 110 & 63 & 1.25 \\
\hline
\end{tabular}

${ }^{\mathrm{a}}$ IDR: indirect digital radiography; ${ }^{\mathrm{b}} \mathrm{DDR}$ : direct digital radiography ; ${ }^{\mathrm{c}} \mathrm{CR}$ : computed radiography; ${ }^{\mathrm{d}}$ Philips Medical Systems, Eindhoven, Netherlands; ${ }^{\mathrm{e}}$ Carestream, New York, United States $;{ }^{\mathrm{f}}$ Siemens Medical Solutions, Munich, German; ${ }^{\mathrm{g}}$ Samsung, Seoul, South Korean; ${ }^{\mathrm{h}}$ SID: source to image detector distance. 